Ann. Sc. forest., 1978, 35 (1), 69-92.

\title{
Sélection juvénile pour la qualité du bois chez certains peupliers noirs
}

\author{
G. NEPVEU*, R. KELLER* ef E. TEISSIER du CROS** \\ * Station de Recherches sur la Qualité des Bois - Centre National de Recherches Forestières \\ Champenoux, 54280 Seichamps \\ **Station d'Amélioration des Arbres Forestiers - Centre de Recherches Forestières d'Orléans \\ Ardon, 45160 Olivet, France
}

\begin{abstract}
Résumé
Vingt-trois clones de Populus nigra L. et vingt-huit de $P$. x. euramericana (Dode) Guinier ont été soumis à des essais destinés à mettre en évidence la possibilité de sélectionner précocement des peupliers pour la qualité de leur bois. Les observations ont porté au stade juvénile sur des morceaux de tiges d'un an écorcées à raison de deux par clone et au stade adulte sur des carottes d'arbres d'une vingtaine d'années de la collection du Populetum National de Vineuil près de Blois (2 par clone également).

L'existence d'une variabilité pour certains caractères offre la possibilité de profiter d'un gain génétique, notamment pour l'infradensité du bois (6 p. 100). Les corrélations génotypiques juvénileadulte pour la densité du bois semblent autoriser une sélection pour ce caractère dès l'âge d'un an. Cela ne conduirait à aucune perte sur la production en volume du fait de l'absence de corrélation densité-croissance. En revanche, cela pourrait se traduire par la sélection indirecte de clones à fort retrait tangentiel.
\end{abstract}

\section{1. - Introduction}

Cet article est la synthèse de deux documents à diffusion limitée, l'un signé Teissier du Cros et Keller (1973) sur la « Détermination précoce de certaines caractéristiques du bois de Populus nigra L. », le deuxième de Nepveu et Teissier du Cros (1976) intitulé : «Sélection juvénile pour la qualité du bois chez les peupliers euraméricains 》. Le premier était surtout une mise au point méthodologique. Le second, profitant de la technique éprouvée au préalable, confirmait les premiers résultats, les complétait et en proposait une interprétation génétique. Ces deux documents comportaient de nombreux graphiques et tableaux, qui ne sont pas tous reproduits ici et auxquels le lecteur pourra se reporter s'il le désire.

La sélection des peupliers se base de plus en plus sur des tris précoces. Mais s'il est déjà possible de bien connaître, dès la pépinière, certains caractères d'adaptation des clones ou leur sensibilité aux maladies, il semblait indispensable pour apprécier 
les caractéristiques de la qualité du bois d'attendre un certain diamètre du tronc, donc un certain temps. Ce temps peut représenter de 8 à 12 ans pour les peupliers. Autant d'années perdues s'il devait apparaître à cette échéance que le bois produit était inutilisable. Nous pouvons donner deux exemples qui démontrent la relation étroite entre la densité du bois de Peuplier et ses qualités mécaniques.

- En Grande-Bretagne, la Société Bryant \& May Lłd produit des allumettes et commence actuellement à s'approvisionner en bois dans ses propres plantations. II y a 20 ans, une partie de ces plantations avait été réalisée avec le clone "Gelrica》 croissant vite sous ces climats. II s'avère maintenant que ce bois, pas assez résistant, est impropre à la confection des bâtons d'allumettes. L'infradensité du bois de ce clone est faible : 0,319 dans les essais anglais (0,339 pour I 214) contre 0,414 pour Robusta $\left({ }^{1}\right)$.

- I 214 est introduit en France au début des années 50. En 1965, le Centre Technique du Bois signale la faible densité de son bois. En 1971, les utilisateurs en découvrent les conséquences : faible résistance des placages déroulés, mauvaise rétention des agrafes. L'infradensité du bois de ce clone est faible : 0,287 dans nos essais contre 0,355 pour Robusta.

La question à laquelle la présente étude se propose de répondre peut donc être libellée comme suit : peut-on sélectionner précocement les peupliers pour la qualité de leur bois?

Pour ce faire, il faut à la fois que les caractères considérés présentent une variabilité génotypique (correspondant non seulement à l'effet additif des gênes, mais également à leurs effets d'interaction - dominance et épistasie — puisqu'il s'agit ici de sélection clonale) à l'état jeune et à l'état adulte, mais aussi que la corrélation génotypique entre les propriétés du bois aux deux âges dont il est question soit significative. Pour répondre à ces questions, il est indispensable de disposer d'un test clonal qui nous permet de décomposer les variances et covariances observées.

En effet, si des corrélations phénotypiques pour des caractères de forme ou de croissance peuvent intéresser un sylviculteur lorsqu'il procède à une éclaircie, l'améliorateur s'intéressera avant tout aux corrélations génétiques et génotypiques juvénile-adulte. Elles seules permettent de prédire qu'une sélection dans le jeune âge aura une efficacité à l'état adulte.

Nous évoquerons donc, dans ce travail, les deux questions suivantes : adulte,

- existence d'une variabilité génotypique tant au stade juvénile qu'au stade

- existence d'une corrélation génotypique jeune adulte.

Nous traiterons également des conséquences d'une sélection de la qualité du bois sur d'autres caractères d'importance économique.

Auparavant, nous éprouverons les différentes méthodologies employées en étudiant la variabilité de deux clones de Peuplier.

( $\left.{ }^{1}\right)$ Résultats aimablement communiqués par Monsieur C. Jilbert de Bryant and May (Forestry) Ltd. 


\section{2. - Matériel}

2.1. - Etude de la variabilité intraclone des caractéristiques de la qualité du bois Type de matériel utilisé.

Carottes radiales de $5 \mathrm{~mm}$ de diamètre prélevées à l'aide d'une tarière à bois tendre, à environ $40 \mathrm{~cm}$ de la base de l'arbre, de façon qu'elles comportent le maximum d'accroissements annuels, tout en évitant la zone du bois plus ou moins déformé par la proximité des grosses racines. On a cherché à atieindre chaque fois le cœur de l'arbre. Au début de chaque prélèvement, le couple de torsion à appliquer sur la tarière a été mesuré, face est.

Lieu de prélèvement.

Populetum de Bourret près de Montauban (82) pour les Populus nigra (*) et Populetum de Vineuil près de Blois (41) pour le peuplier euraméricain.

Clones:

à Bourret: P. nigra, mâle présumé «Blanc de Garonne » vingt-quatre arbres répartis en deux blocs

à Vineuil : P. x euramericana, cv «Robusta » vingt-huit arbres répartis en huit blocs.

Direction de prélèvement.

Une carotte vers le vent dominant (ovest), une carotte sous le vent dominant (est) ou encore une seule carotte traversant le tronc de part en part, ensuite coupée en deux à la moelle.

2.2. - Etude de la variabilité génotypique des caractéristiques de la qualité du bois

- Arbres adultes :

- Type de matériel étudié : mêmes caractéristiques qu'au paragraphe 21.

- Lieu de prélèvement : Populetum de Vineuil.

- Clones : 23 clones de P. nigra à raison de deux arbres par clone

28 clones de P. $x$ euramericana à raison de deux arbres par clone $(* *)$.

- Pousses d'un an :

- Type de matériel utilisé : partie de tige comprise entre 5 et $15 \mathrm{~cm}$ de la base.

- Lieu de prélèvement : pépinière de Velaine près de Nancy (54).

- Clones : comme pour les arbres adultes.

- Nombre d'échantillons par clone : 2.

Soulignons dès à présent le nombre faible d'individus par clone : 2 .

(*) Aimablement mis à notre disposition par le Centre Technique du Génie Rural des Eaux et des Forêts, pour cette étude.

$(* *)$ Voir en annexe la liste des $P$. nigra, leur origine et la liste des peupliers euramericains, ainsi que les abréviations employées sur les figures. 


\section{3. - Caractères étudiés et méthodologie}

\section{1. - Infradensité}

\subsection{Parties de tige d'un an.}

L'infradensité d'un échantillon est le rapport de son poids anhydre à son volume saturé. Le poids anhydre est obtenu après séchage à l'étuve à $102^{\circ} \mathrm{C}$ pendant $24 \mathrm{~h}$. Le volume saturé est mesuré lorsque le point de saturation des fibres est atteint, c'est-àdire lorsque le taux d'humidité de l'échantillon dépasse 30 p. 100 environ. Ce taux est atteint par immersion $48 \mathrm{~h}$ dans l'eau.

La mesure de l'infradensité pour les tiges de P. nigra a été réalisée de deux façons :

a) Au voluménomètre.

L'infradensité est alors obtenue directement par le rapport

$$
\frac{\text { poids anhydre }}{\text { volume saturé }}
$$

b) Par la saturation intégrale.

Cette méthode découverte par Keylwerth en 1954 et adaptée par Polge (1963) pour la mesure de l'infradensité sur des carottes prélevées à la tarière de Pressler est maintenant utilisée couramment ; la formule de travail est :

$$
\frac{\mathrm{PO}}{\mathrm{VS}}=\frac{1}{\frac{\mathrm{PM}}{\mathrm{PO}}-0,347}
$$

$\mathrm{PO}=$ poids anhydre après $24 \mathrm{~h}$ à $102^{\circ}$

$\mathrm{VS}=$ volume saturé

$\mathrm{PM}=$ poids maximum de l'échantillon intégralement saturé d'eau par ébullition durant $48 \mathrm{~h}$.

$$
0,347=1-\frac{1}{1,53}
$$

où $1,53=$ densité de la matière ligneuse proprement dite.

Dans ce cas, le rapport $\frac{P O}{V S}$ est donc obtenu indirectement grâce aux pesées de PM et de PO. Les deux méthodes essayées sur Populus nigra ont donné des valeurs concordantes $(r=0,90 * *)$ qui ont permis de n'estimer les infradensités des pousses d'un an des peupliers euraméricains qu'avec la méthode de saturation intégrale, méthode la plus rapide des deux.

\subsection{Carottes d'arbres adultes.}

Les infradensités, ainsi d'ailleurs que les autres caractères mesurables directement sur carottes (retraits), ont été estimés sur $3 \mathrm{~cm}$ de chaque extrémité de ces échantillons. Pour chaque carotte, précisons qu'une extrémité est constituée de bois formé 
pendant le stade juvénile des plants (bois côté moelle), l'autre correspondant à ses cernes adultes (bois sous écorce ou périphérique).

La méthode employée est celle de la saturation intégrale. Pour les peupliers euraméricains, elle a été réalisée deux fois pour chaque échantillon, c'est-à-dire avant et après dissolution des cristaux de carbonate de calcium. En effet Teissier du Cros et Keller (op. cit.) ont constaté chez Populus nigra une corrélation négative et fortement significative entre les infradensités des cernes juvéniles et des cernes périphériques ( $r=-0,89 * *$ pour l'un des blocs du Populetum de Bourret, $r=-0,54 * *$ pour les deux blocs - figure 1). Cette liaison devient non significative après élimination des points aberrants. Polge (1973) a montré que ces points rentraient dans la norme après élimination des cristaux. Cette dernière a été obtenue en faisant séjourner les échantillons dans une solution d'acide chlorhydrique $0,1 \mathrm{~N}$ pendant 4 jours à froid et sous vide.

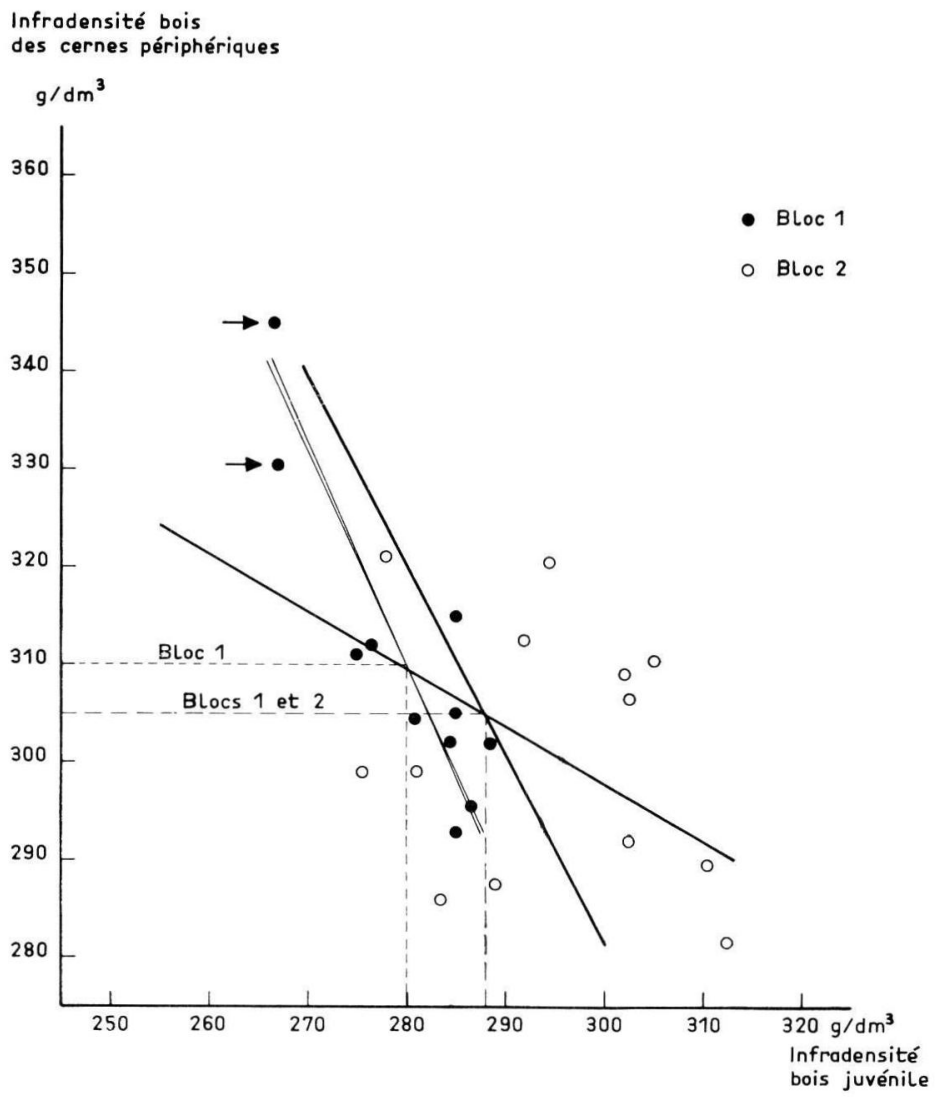

FIG. 1. - Régression entre infradensité du bois des cernes périphériques et celle du bois juvénile chez Populus nigra.

Regression between peripheric and central rings wood basic densities. 


\section{2. - Couple de torsion}

Le couple de torsion nécessaire à la pénétration des tarières a été mesuré systématiquement du côté sous le vent au moment du prélèvement des carottes ; la méthode employée est celle décrite par Polge et Keller (1970) ; le caractère est exprimé en $\mathrm{cm}$. $\mathrm{kg}$.

\section{3. - Retraits}

Les retraits correspondent à la différence des dimensions axiale, tangentielle et radiale entre l'état saturé ef l'état sec à l'air des échantillons, fixé à un taux d'humidité de 10 p. 100, voisin de celui du bois mis en œuvre.

Les retraits axial ef tangentiel sont mesurés avec un micromètre à lecture semiautomatique constitué par un capteur électronique de déplacement couplé à un télétype selon une méthode décrite par Polge, Keller et Thiercelin (1973) ; un avancement progressif de la carotte permet une mesure tous les deux millimètres environ. Les résultats que l'on utilise sont des moyennes d'une soixantaine d'observations. Le retrait radial est mesuré sur la longueur totale de l'échantillon avec un micromètre à tare. Les retraits sont tous exprimés en pourcentage des dimensions à 10 p. 100 d'humidité.

\section{4. - Longueur moyenne des fibres}

Sur chaque éprouvette de $3 \mathrm{~cm}$, la longueur moyenne des fibres a été estimée à l'aide d'une dizaine d'entre elles (voir Nepveu et Teissier du Cros 1976 op. cit. pour l'échantillonnage) ; un échantillon a été coloré à la safranine, étalé sur une coupe microscopique qui projetée, sur un écran, permet des mesures grâce à un curvimètre électronique relié à un télétype. Les fibres doivent être intactes, le choix au hasard doit être respecté.

Cette observation n'a été réalisée que sur P. $x$ euramericana.

\section{5. - Rendement en fibres}

Il est estimé par le rapport poids de fibres à l'état anhydre sur poids anhydre de bois. Ełant donné la masse trop faible des échantillons correspondant aux extrémités de carottes, ce paramètre a été mesuré sur ce qui restait des carottes entre les extrémités cœur et écorce. II ne pourra pas répondre à certaines de nos préoccupations qui sont les liaisons jeune-adulte au moyen de mesures faites sur les extrémités d'une même carotte.

Ce même rendement a été mesuré sur les tiges d'un an.

Les conditions de cuisson étaient les suivantes :

- taux d'alcali actif : 16 p. 100

- rapport liquide sur bois : 4

- sulfidité : 25 p. 100

- cuisson Kraft

- temps de palier : $30 \mathrm{mn}$

- température de palier : $170^{\circ} \mathrm{C}$. 
Comme pour la longueur de fibres, ce caractère n'a été mesuré que chez les peu. pliers euraméricains.

\section{6. - Croissance}

Chaque fois que cela a été nécessaire, la qualité du bois a été reliée à des données auxométriques réalisées tout au long de la vie des arbres étudiés.

Il faut noter que, surtout dans le jeune âge, les pousses en hauteur et les croissances en circonférence sont souvent à considérer avec prudence du fait que les années d'installation diffèrent suivant les arbres; ceci ne doit pas être sans influence sur la croissance initiale. Il est à craindre également que les conditions de plantation n'aient pas été identiques.

\section{7. - Remarque importante}

Les clones de la collection de Vineuil sont généralement installés en parcelles unitaires de deux à cinq arbres sans répétition. Les deux arbres échantillonnés par clone ont donc vécu dans des conditions très proches. Les variances intraclones risquent fort d'être sous-estimées relativement aux variances interclones (effet d'environnement commun) ; ceci implique une possibilité de surestimation des héritabilités au sens large. Il en est de même pour les échantillons de bois d'un an prélevés sur deux pieds-mères proches, sur deux tiges d'un même pied-mère ou même dans un cas sur la même tige.

\section{4. - Résultats}

\section{1. - Etude de la variabilité intraclone des caractéristiques de la qualité du bois}

Les seules causes de variation décelables ici sont naturellement non génétiques ; elles sont normalement dues aux effets du milieu. Ceux-ci se décomposent en :

- effets des blocs, qui englobent les conditions d'élevage, la grosseur et l'âge des plants à l'installation, la fertilité des blocs ;

- effets de l'orientement par rapport aux vents dominants;

- effet de l'âge du bois, c'est-à-dire les variations observées entre le bois de cœur et le bois périphérique ;

- effets des cristaux, c'est-à-dire principalement la variation des caractéristiques avant et après enlèvement des cristaux.

Ces effets sont analysés pour chacun des caractères étudiés.

\subsection{Densité du bois.}

Seul Populus nigra accuse une variation de densité que l'on peut attribuer à l'effet bloc (tabl. 1). Cette différence ne se manifeste statistiquement qu'au stade juvénile, la plus forte densité étant obtenue là où la circonférence à 6 ans était la plus faible : $58,4 \mathrm{~cm}$ en moyenne pour le bloc 2 contre $70,5 \mathrm{~cm}$ pour le bloc 1 . A l'état adulte le classement des infradensités des arbres s'inverse par rapport au classement juvénile, 
mais la différence n'est plus significative. D'ailleurs, les accroissements cumulés de la $15^{\mathrm{e}}$ à la 19e année ne diffèrent pas d'un bloc à l'autre.

\section{TABLEAU 1}

Effet bloc sur la densité du bois de Populus nigra «Blanc de Garonne »

Block effect on the wood density of P. nigra "Blanc de Garonne »

\begin{tabular}{|c|c|c|c|c|}
\hline & & $\begin{array}{c}\text { Bloc } 1 \\
\text { (11 arbres) }\end{array}$ & $\begin{array}{c}\text { Bloc } 2 \\
\text { (13 arbres) }\end{array}$ & Test de Student \\
\hline $\begin{array}{c}\text { Infradensité } \\
\text { du bois }\end{array}$ & Côté moelle .... & 280 & 295 & significatif à 1 p. 100 \\
\hline en $\mathrm{g} / \mathrm{dm}^{3}$ & Côté écorce . . . . . & 310 & 301 & non significatif \\
\hline
\end{tabular}

L'orientement n'affecte pas la densité des Populus nigra de Bourret; en revanche, il a une légère influence pour les Robusta de Vineuil en donnant une supériorité (significative à 5 p. 100) de la densité sur la face au vent des arbres. Cela se traduit vraisemblablement par la production de bois de tension sur cette face. Cette caractéristique est connue chez Robusta, qui a souvent tendance à produire du bois «pelucheux" (Centre Technique du Bois, 1957).

La densité du bois augmente avec l'âge des arbres, tout au moins pour les deux clones que nous avons étudiés (tabl. 2).

TABLEAU 2

Variation de l'infradensité avec l'âge

Age effect on the density

\begin{tabular}{|c|c|c|c|c|}
\hline \multicolumn{2}{|c|}{ Densité du bois en $\mathrm{g} / \mathrm{dm}^{3}$} & \multirow{2}{*}{$\begin{array}{c}\text { Moelle } \\
280\end{array}$} & \multirow{2}{*}{$\begin{array}{l}\text { Ecorce } \\
310\end{array}$} & \multirow{2}{*}{$\begin{array}{c}\text { Test statistique } \\
\text { significatif à } 1 \text { p. } 100\end{array}$} \\
\hline Blanc de ) & Bloc $1 \ldots \ldots \ldots \ldots$ & & & \\
\hline Garonne & Bloc $2 \ldots \ldots \ldots$ & 295 & 301 & non significatif \\
\hline Robusta & Moyenne est-ouest . . & 336 & 376 & significatif à 1 p. 100 \\
\hline
\end{tabular}

Cette augmentation est quasi générale chez les espèces forestières; elle concorde avec les observations de Polge (1964).

Le traitement à l'acide chlorhydrique, réalisé seul sur Robusta, se traduit par une diminution de 3 p. 1000 de l'infradensité, signe que la teneur en cristaux de carbonate de calcium de ce clone est faible.

\subsection{Retraits.}

Chez "Blanc de Garonne » comme chez «Robusta», seul le retrait tangentiel semble affecté par l'âge du bois (tabl. 3). 
TABLEAU 3

Variation du retrait tangentiel avec l'âge du bois

Age effect on tangentiel shrinkage

\begin{tabular}{lccc}
\hline Retrait tangentiel en p. 100 & Moelle & Ecorce & Test statistique \\
\hline Blanc de Garonne $\ldots \ldots \ldots \ldots$ & 6,78 & 7,18 & significatif à 5 p. 100 \\
Robusta $\ldots \ldots \ldots \ldots \ldots \ldots$ & 7,33 & 6,89 & significatif à 1 p. 100 \\
\hline
\end{tabular}

Mais cette variation se fait en sens contraire pour les deux clones. Alors que Blanc de Garonne semble réagir classiquement (voir Polge 1964, op. cit.), Robusta a une réaction inhabituelle.

\subsection{Longueur des fibres.}

Ce caractère a été mesuré chez Robusta seulement. Du centre vers la périphérie, la longueur varie de 1,13 à $1,43 \mathrm{~mm}$, phénomène habituel chez les essences ligneuses, ne militant pas en faveur des très courtes rotations pour la production de pâte à papier.

\subsection{Rendement en fibres.}

Rappelons que ce caractère aussi n'a été mesuré que chez Robusta. De plus nous n'avons pas pu observer les variations dues à l'âge du bois. Ce rendement atteint 58,04 p. 100 à l'est contre 56,76 à l'ouest. Nous trouvons là une certc ine analogie avec une étude de Janin (1973) qui note pour le bois de réaction du pin maritime un rendement notablement plus faible que pour celui du bois normal. Cependant, le bois formé du côté ouest des tiges ne peut pas être considéré comme un bois de tension typique : même si la densité est légèrement plus forte, aucune différence n'apparaît dans les retraits.

\section{2. - Variations interclones des caractéristiques du bois chez Populus nigra et Populus euramericana}

Les effets que nous cherchons à mettre en évidence ici sont surtout génolypiques. L'analyse tient compte, en outre, des effets déjà étudiés au paragraphe 4.1, à savoir :

- les effets de l'orientement,

- les effets de l'âge du bois,

- l'influence des cristaux.

4.21. Densité du bois.

4.211. Effet clone.

Les figures 2 et 3 donnent les valeurs des moyennes est-ouest des infradensités pour les différents clones étudiés. On note un effet «clone » très marqué qui amène plusieurs remarques: 
- cette variabilité se manifeste aussi bien à l'état juvénile qu'à l'état adulte ;

- chez Populus nigra, elle se traduit par une amplitude totale entre la plus faible densité côté écorce et la plus forte de 23 p. 100 et entre la plus forte et la moyenne de 12 p. 100 ;

- chez Populus euramericana, elle est de 24 p. 100 entre le clone à plus forte densité (Robusta) et celui dont la densité est la plus faible (1 214). Robusta a une densité qui dépasse de 9 p. 100 la densité moyenne des clones étudiés. L'héritabilité au sens large de cette densité est de 0,71 (†abl. 8). Le gain génotypique relatif obtenu en sélectionnant Robusta pour sa densité est de $G=9 \times 0,71=6,4$ p. 100 . Cette valeur peut paraître faible. Elle est due essentiellement à la faible amplitude de notre échantillon. II n'est pas improbable qu'on puisse augmenter artificiellement cette variabilité par croisements contrôlés entre espèces Populus nigra et Populus deltoïdes chez qui une sélec-

Bois juvénile
Côté moëlle

(Moyenne Est-Ouest)

$\mathrm{g} / \mathrm{dm}^{3}$

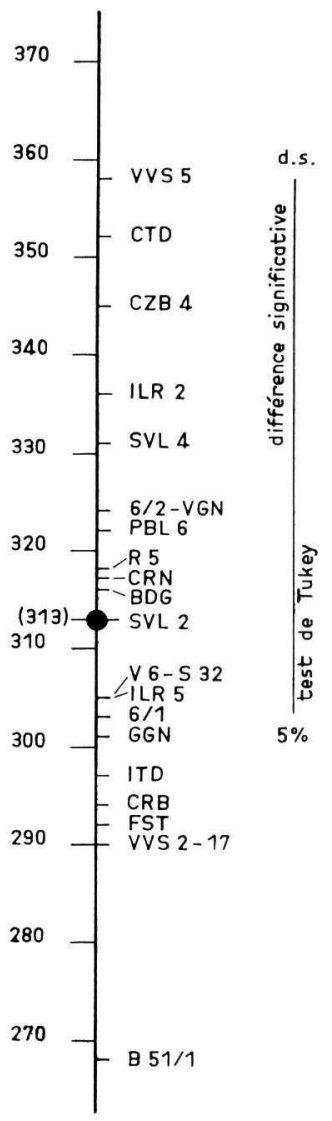

Bois d'un an

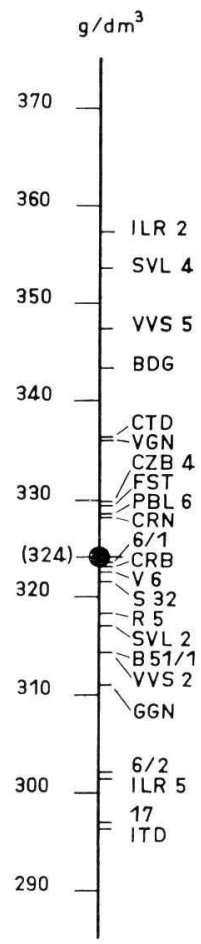

Bois adulte

Côté écorce

(Moyenne Est-Ovest) $\mathrm{g} / \mathrm{dm}^{3}$

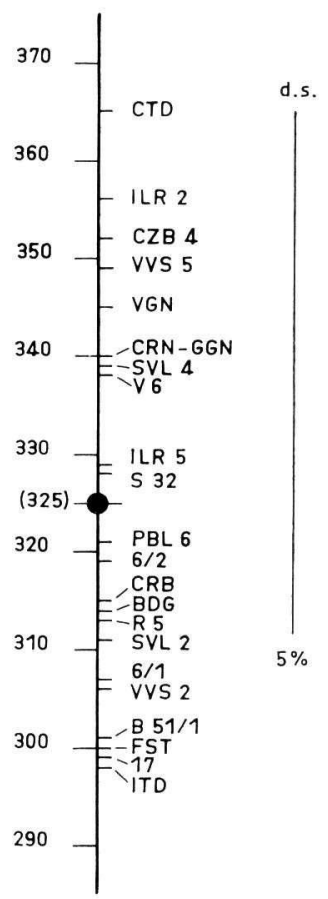

FIG. 2. - Infradensité. Comparaison des moyennes de clones : Populus nigra.

Basic density. Comparison of clone means : Populus nigra. 
Bois juvénile

Côté moëlle (Moyenne Est-Ouest) $\mathrm{g} / \mathrm{dm}^{3}$

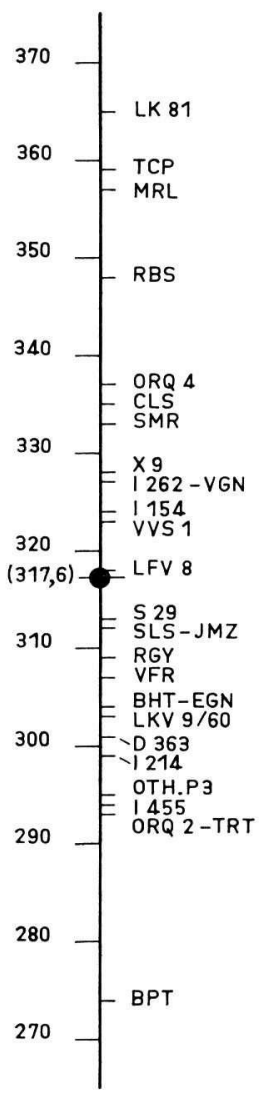

Bois d'un on

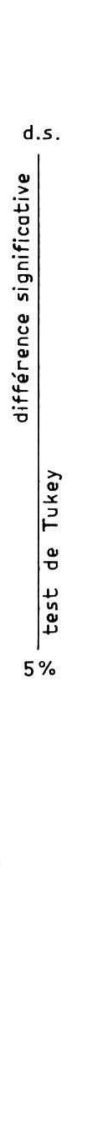

Bois odulte

Côté écorce

(Moyenne Est-Ouest)

$$
\mathrm{g} / \mathrm{dm}^{3}
$$

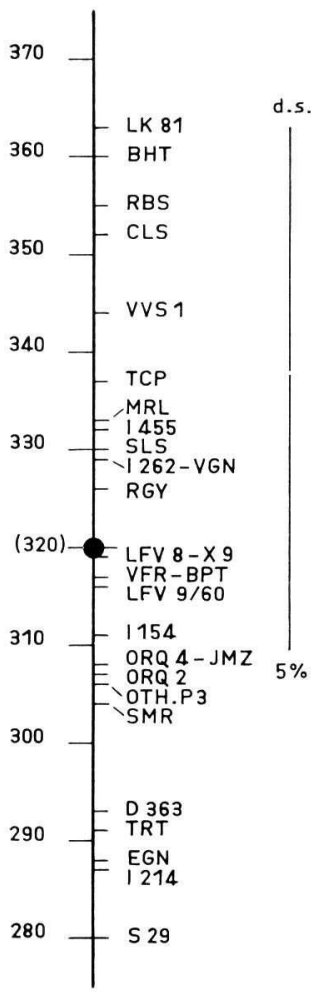

FIG. 3. - Infradensité. Comparaison des moyennes de clones : Populus euramericana.

Basic density. Comparison of clone means : Populus euramericana.

tion préalable pour une forte densité du bois serait réalisée $\left({ }^{1}\right)$. Remarquons toutefois qu'il ne faut pas négliger un progrès génotypique de 6 p. 100 sur la densité : en effet, son poids économique pour certaines utilisations peut être plus considérable qu'une amélioration de 20 ou 30 p. 100 pour une autre caractéristique. Des travaux dans ce sens devraient être engagés afin de chiffrer les gains financiers à attendre de la sélection caractère par caractère et pour chaque emploi du matériau.

\subsection{Effet de l'orientement.}

Le tableau 4 montre les effets des vents dominants d'ouest. Ils ne sont pas significatifs chez Populus nigra, mais le sont manifestement pour les peupliers euraméricains.

(1) Dans l'optique où une forte densité du bois des peupliers est un caractère effectivement recherchó. 


\section{TABLEAU 4}

Effet de l'orientement sur la densité du bois

Orienting effect on the wood density

\begin{tabular}{|c|c|c|c|c|}
\hline Espèce & $\begin{array}{l}\text { Infradensité } \\
\text { en } \mathrm{g} / \mathrm{dm}^{3}\end{array}$ & $\begin{array}{c}\text { Face au } \\
\text { vent } \\
\text { (ouest) }\end{array}$ & $\begin{array}{c}\text { Face sous } \\
\text { le vent } \\
\text { (est) }\end{array}$ & Résultats des tests statistiques \\
\hline Populus nigra & $\begin{array}{l}\text { côté moelle } \\
\text { côté écorce }\end{array}$ & $\begin{array}{l}317 \\
330\end{array}$ & $\begin{array}{l}309 \\
321\end{array}$ & $\left.\begin{array}{l}\text { non significatif } \\
\text { non significatif }\end{array}\right\} \begin{array}{l}\text { test de Student } \\
\text { à } 90 \mathrm{D} . \mathrm{D} . \mathrm{L} .\end{array}$ \\
\hline $\begin{array}{l}\text { Populus } \\
\text { euramericana }\end{array}$ & $\begin{array}{l}\text { côté moelle } . \\
\text { côté écorce } .\end{array}$ & $\begin{array}{l}322 \\
327\end{array}$ & $\begin{array}{l}313 \\
314\end{array}$ & $\left.\begin{array}{l}\text { hautement significatif } \\
\text { hautement significatif }\end{array}\right\} \begin{array}{l}\text { test F à } 1 \\
\text { et } 224 \text { D. D. L. }\end{array}$ \\
\hline
\end{tabular}

L'interaction clone $x$ orientement hautement significative chez ces hybrides montre que les différents génotypes ne se comportent pas de la même manière. Ceci peut être illustré par les valeurs de quelques clones fréquemment plantés en France ou utilisés dans les Populetum expérimentaux (tabl. 5). Dans ce cas précis, il s'agit d'arbres installés dans le Populetum de Vineuil et d'un bois situé du côté des cernes périphériques.

\section{TABLEAU 5}

L'anisotropie du bois chez quelques peupliers euraméricains

The wood anisotropy in a P. euramericana clones sample

\begin{tabular}{|c|c|c|c|}
\hline \multirow{2}{*}{ Clones } & \multicolumn{2}{|c|}{ Infradensité en $\mathrm{g} / \mathrm{dm}^{3}$} & \multirow{2}{*}{ Différences } \\
\hline & Est & Ouest & \\
\hline 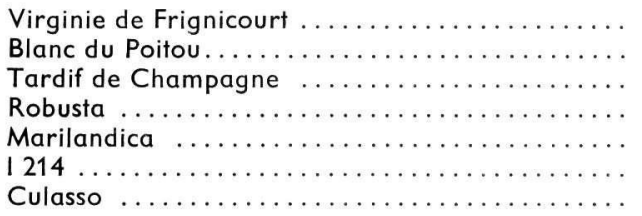 & $\begin{array}{l}292 \\
292 \\
327 \\
355 \\
333 \\
292 \\
360\end{array}$ & $\begin{array}{l}343 \\
342 \\
347 \\
358 \\
333 \\
282 \\
343\end{array}$ & $\begin{array}{r}+51 \\
+50 \\
+20 \\
+\quad 3 \\
-10 \\
-17\end{array}$ \\
\hline
\end{tabular}

II ne faut pas oublier, au cours de la lecture de ce tableau, un fait important : l'absence de dispositif sur le terrain. Ceci peut expliquer que certains clones installés en un endroit du Populetum peu protégé des vents dominants présentaient une anisotropie à la différence d'autres, moins exposés.

\subsection{Influence de l'âge du bois.}

Contrairement à ce qui se passe normalement chez les espèces étudiées jusqu'ici, les peupliers n'accusent pas systématiquement une augmentation de la densité de leur bois du cœur vers la périphérie de l'arbre. Si, en moyenne, la valeur de l'extérieur est supérieure, chez Populus nigra (313 et $325 \mathrm{~g} / \mathrm{dm}^{3}$ ), elle n'est pas significativement différente et, chez Populus euramericana (318 et $321 \mathrm{~g} / \mathrm{dm}^{3}$ ), cette différence est assortie 
d'une interaction clone-âge significative qui est la conséquence d'une absence de loi générale de variation de cette densité avec l'âge des arbres.

\subsection{Influence des cristaux.}

L'analyse de variance fait ressortir un effet significatif de l'enlèvement des cristaux de carbonate de calcium qui confirme les travaux de Clément et Janin (1972). Rappelons que cet effet avait été très marqué chez deux individus du clone Blanc de Garonne. La répartition de ces cristaux n'apparaît pas homogène d'une partie à l'autre du tronc et d'un clone à l'autre. On constate en effet sur le tableau 6 que le traitement a été en moyenne plus efficace sur la face est du cœur des arbres que sur la face ouest, mais l'analyse statistique ne révèle aucune interaction «cristauxorientement ». Par ailleurs, ce même tableau permet d'approcher la répartition des cristaux dans l'arbre en fonction de l'âge des cernes. Les cristaux seraient plus concentrés vers la périphérie du fût (interaction «cristaux-âge » significative). Cette conclusion ne semble pas concorder tout à fait avec celle de l'étude de Clément et Janin.

\section{TABLEAU 6}

Effet du traitement contre les cristaux

Effect of the treatment against calcium carbonate cristals

\begin{tabular}{|c|c|c|c|}
\hline \multirow{2}{*}{ Parties de carottes analysées } & \multicolumn{2}{|c|}{ Infradensité en $\mathrm{g} / \mathrm{dm}^{3}$} & \multirow{2}{*}{ Différence } \\
\hline & avant traitement & après traitement & \\
\hline 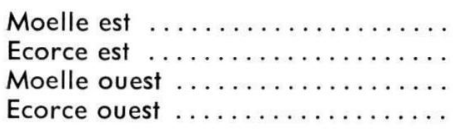 & $\begin{array}{l}314 \\
327 \\
328 \\
335\end{array}$ & $\begin{array}{l}313 \\
314 \\
322 \\
327\end{array}$ & $\begin{array}{l}-1 \\
-13 \\
-6 \\
-8\end{array}$ \\
\hline
\end{tabular}

\subsection{Retraits.}

Pour Populus nigra comme pour Populus $x$ euramericana seul le retrait tangentiel accuse une variabilité génotypique dont l'amplitude semble légèrement plus élevée chez la première espèce que chez la seconde. Dans leur ensemble, ces valeurs restent relativement faibles puisqu'elles ne dépassent pas 8,5 p. 100 pour $P$. nigra (Piboulette 6) et 8,2 p. 100 pour les euraméricains (Culasso).

L'analyse des autres effets montre principalement une diminution de la valeur du retrait tangentiel du cœur vers la périphérie du tronc, comme le montre le tableau 7.

TABLEAU 7

Effet de l'âge du bois sur la valeur du retrait tangentiel

Age effect on tangentiel shrinkage

\begin{tabular}{lcc}
\hline Retrait en p. 100 & Cœur & Périphérie \\
\hline Populus nigra ........ & 7,2 & 6,9 \\
Populus x euramericana. & 9,2 & 7,3 \\
\hline
\end{tabular}




\subsection{Longueur des fibres.}

Sur les pousses d'un an cette longueur est très faible, 0,69 mm en moyenne, et ne semble pas dépendre du facteur génotypique. Lorsqu'on passe au bois de cœur, cette valeur atteint déjà $1 \mathrm{~mm}$. L'échantillon «bois de cœur » concerne en effet les deux ou trois premiers cernes. On assiste donc à une augmentation rapide de cette longueur, qui se poursuit jusqu'aux cernes périphériques du tronc : $1,35 \mathrm{~mm}$ en moyenne. On discernera à ce stade une certaine variabilité génotypique, assortie d'une héritabilité au sens large de 0,47 qui permettrait un gain génétique en sélectionnant à l'état adulte le clone présentant les fibres les plus longues : 1,44 mm (Tardif de Champagne) par rapport à une moyenne de $1,35 \mathrm{~mm}$ soit 6,7 p. 100 :

$$
\mathrm{G}=6,7 \times 0,47=3 \text { p. } 100 .
$$

Ce gain, faible, mais vraisemblablement calculé sur un échantillonnage de clones trop restreint, ne semble pas encourager à prendre en compte le caractère « longueur de fibres » dans un programme de sélection.

Une autre conclusion, à notre avis importante, de ce paragraphe est que la pratique de courtes rotations pour la production de bois de pâte à papier n'est sûrement pas une voie avantageuse. Mais la brièveté des fibres au cœur de l'arbre devrait inciter à rechercher cerne par cerne la variation de leur longueur, étude qui permettrait de déterminer à partir de quel âge les peupliers euraméricains donnent des fibres dont la longueur serait proche de celle de fibres produites dans les cernes périphériques d'arbres adultes.

\subsection{Rendement en fibres.}

Sur les pousses d'un an comme pour les arbres de Vineuil, ce caractère semble soumis à une certaine variabilité génotypique ; mais comme pour la longueur des fibres, l'héritabilité reste assez faible (0,59 pour les pousses d'un an et 0,42 pour les arbres adultes) ; elle ne permettrait pas un gain génétique important ( 2 p. 100) et ne justifierait certainement pas une prise en compte de ce caractère dans un programme de sélection.

Ce caractère est aussi soumis à un effet significatif de l'orientement (est 56,05 p. 100 , ovest 58,22 p. 100) qui est en opposition avec les résultats rencontrés avec Robusta. Aucune interaction «clone $x$ orientement » n'a pourtant été décelée.

\subsection{Héritabilités.}

Nous avons, pour quelques-uns des caractères étudiés, mentionné des valeurs d'héritabilité au sens large. Leur calcul supposait la réalisation d'un certain nombre d'hypothèses, notamment le fait que nous devions avoir affaire à un échantillon de clones pris au hasard. Les clones du Populetum de Vineuil ont tous été sélectionnés pour « quelque chose ». La rectitude du fût, la grosseur du tronc et un accroissement fort en hauteur ont généralement été les caractères retenus. Ceci aura pour effet d'amoindrir considérablement l'intérêt des héritabilités calculées sur les caractères auxométriques puisque nous ne disposons plus d'un échantillonnage de la population initiale. En outre, les corrélations génotypiques densité-croissance que nous calcule- 
rons seront elles-mêmes biaisées et ne pourront donc nous apprendre si, pour la densité, l'éventail des clones dont nous disposons est représentatif de l'espèce étudiée.

Deux ramets étudiés par ortet est certainement un nombre trop faible; mais pis, l'absence de répétitions suscepłible de provoquer un amenuisement de la variance intraclone, relativement à la variance interclone (effet d'environnement commun) peut conduire à une surestimation de l'héritabilité.

Pour toutes les raisons évoquées ci-dessus, les résultats présentés doivent être considérés comme de simples indications. Le tableau 8 donne quelques exemples d'où il ressort une forte héritabilité des caractères de croissance, une héritabilité assez élevée de la densité et d'un caractère qui lui est généralement étroitement lié : le couple de torsion, des héritabilités plutôt faibles des autres caractères.

\section{TABLEAU 8}

Héritabilités au sens large

Broad sense heritabilities

\begin{tabular}{|c|c|c|c|}
\hline & \multirow{2}{*}{ Caractères } & \multicolumn{2}{|c|}{ Héritabilité au sens large } \\
\hline & & P. euramericana & P. nigra \\
\hline \multicolumn{4}{|l|}{ Densités } \\
\hline \multicolumn{2}{|c|}{ pousses d'un an } & $0,71 * *$ & - \\
\hline \multicolumn{2}{|c|}{ arbres adultes $\left({ }^{1}\right)\left\{\begin{array}{l}\text { côté cœur } \ldots \ldots \ldots \ldots \ldots \\
\text { côté écorce......... }\end{array}\right.$} & $\begin{array}{l}0,55^{* *} \text { à } 0,65 * * \\
0,39 * \text { à } 0,72 * *\end{array}$ & $\begin{array}{l}0,61 * * \\
0,69 * *\end{array}$ \\
\hline \multicolumn{2}{|l|}{ Couple de torsion } & $0,79 * *$ & $0,78 * *$ \\
\hline \multicolumn{4}{|l|}{ Retraits } \\
\hline tangentiel $\left({ }^{1}\right)$ & $\begin{array}{l}\text { côté cœur } \ldots \ldots \ldots \ldots \ldots \ldots \ldots \\
\text { côté écorce } \ldots \ldots \ldots \ldots \ldots \ldots\end{array}$ & $\begin{array}{l}0,05 \text { NS à } 0,47^{* *} \\
0,00 \quad \text { à } 0,32 *\end{array}$ & $\begin{array}{l}0,67 * * \\
0,59 * *\end{array}$ \\
\hline radial $\left({ }^{1}\right)$ & $\begin{array}{l}\text { côté cœur } \ldots \ldots \ldots \ldots \ldots \ldots \ldots \\
\text { côté écorce } \ldots \ldots \ldots \ldots \ldots \ldots\end{array}$ & $\begin{array}{l}0,11 \text { NS à } 0,13 \text { NS } \\
0,05 \text { NS à } 0,22 \text { NS }\end{array}$ & $\begin{array}{l}0,03 \mathrm{NS} \\
0,19 \mathrm{NS}\end{array}$ \\
\hline axial $\left({ }^{1}\right)$ & $\begin{array}{l}\text { côté cœur } \ldots \ldots \ldots \ldots \ldots \ldots \ldots \\
\text { côté écorce } \ldots \ldots \ldots \ldots \ldots \ldots\end{array}$ & $\begin{array}{l}0,00 \text { à } 0,38^{*} \\
0,11 \text { NS à } 0,33^{*}\end{array}$ & $\begin{array}{l}0,02 \text { NS } \\
0,43^{*}\end{array}$ \\
\hline \multicolumn{4}{|l|}{ Longueur des fibres } \\
\hline $\begin{array}{l}\text { bois d'un an } \\
\text { arbres adultes }\end{array}$ & $\left\{\begin{array}{l}\text { côté cœur } \ldots \ldots \ldots \ldots \ldots \ldots \\
\text { côté écorce } \ldots \ldots \ldots \ldots \ldots\end{array}\right.$ & $\begin{array}{c}0,27 \text { NS } \\
0,11 \text { NS à } 0,32 * \\
0,19 \text { NS à } 0,35^{*}\end{array}$ & $\frac{-}{-}$ \\
\hline \multicolumn{4}{|l|}{ Rendement en fibres } \\
\hline \multicolumn{2}{|c|}{$\begin{array}{l}\text { bois d'un an } \\
\text { arbres adultes }\left({ }^{2}\right)\end{array}$} & $\begin{array}{l}0,59 * * \\
0,42 *\end{array}$ & - \\
\hline \multicolumn{4}{|l|}{ Acroissement } \\
\hline hauteur & $\begin{array}{l}\text { jeune } \ldots \ldots \ldots \ldots \ldots \ldots \ldots \ldots \\
\text { adulte } \ldots \ldots \ldots \ldots \ldots \ldots\end{array}$ & $\begin{array}{l}0,84 * * \\
0,80 * *\end{array}$ & $\begin{array}{l}0,66 * * \\
0,60 * *\end{array}$ \\
\hline circonférence & $\begin{array}{l}\text { jeune } \ldots \ldots \ldots \ldots \ldots \ldots \ldots \ldots \ldots \ldots \ldots \\
\text { adulte } \ldots \ldots \ldots \ldots \ldots \ldots\end{array}$ & $\begin{array}{l}0,82 * * \\
0,90 * *\end{array}$ & $\begin{array}{l}0,63 * * \\
0,82 * *\end{array}$ \\
\hline
\end{tabular}

* Significatif à 5 p. 100, ** Significatif à 1 p. 100, NS non significatif.

(1) Selon la localisation des échantillons prélevés (est ou ouest).

(2) Sur demi-carotte privée des extrémités cœur et écorce 
4.3. - Corrélations génotypiques juvénile-adulte ; stabilité du classement des clones avec l'âge

L'estimation de ces paramètres peut être réalisée de deux manières :

4.31. Par régression entre moyennes de clone pour le bois des pousses d'un an et celui des cernes périphériques des arbres adultes.

Nous obtenons une covariance génotypique jeune-adulte, au sens large. En effet, les milieux étant tout à fait différents, les covariances dues à l'environnement sont nulles ; les ressemblances entre les classements des clones à l'état jeune et à l'état adulte ne sont dues qu'à des causes génétiques. Cette covariance divisée par le produit des écarts-types génotypiques estimés lors des calculs des héritabilités au sens large, conduit au coefficient de corrélation recherché.

C'est la corrélation génotypique jeune-adulte qui correspond exactement aux aspects pratiques de la sélection que nous serions amenés à faire : sélection sur des pousses d'un an en pépinière.

4.32. Par analyse de variance-covariance dans les clones entre le bois côté moelle et le bois périphérique des mêmes arbres adultes.

Nous obtenons cette fois encore une corrélation génotypique mais également la corrélation due au milieu et par combinaison la corrélation phénotypique. On pourrait nous faire un reproche concernant notre qualificatif de "juvénile » pour le bois côté cœur. En effet, il est probable que les caractéristiques physiques ou chimiques du bois de cœur des arbres adultes ne sont plus les mêmes que lorsque ces arbres avaient quelques années seulement. Les processus de duraminisation qui suivent la croissance de l'arbre modifient ses caractéristiques. Il serait assez étonnant cependant que ces phénomènes puissent altérer le sens de la corrélation génotypique observée.

Les tableaux 9 et 10 rassemblent les principales valeurs des coefficients de corrélation génotypique juvénile-adulte. Seuls ont été calculés les coefficients correspondant à des caractéristiques fortement héritables de la qualité du bois.

TABLEAU 9

Corrélations génotypiques juvénile-adulte

Juvenile mature genotypic correlations

Entre les caractères

P. nigra

P. euramericana

Densité du bois de pousses d'un an et densité du bois

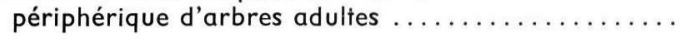

$0,72 * * \quad 0,60 * *$

Longueur des fibres de pousses d'un an et longueur des fibres des cernes périphériques d'arbres adultes ..... 


\section{TABLEAU 10}

Corrélations génotypiques pour la densité du bois d'arbres adultes entre leur centre et leur périphérie

Mature trees wood density genotypic correlations between the center and the periphery of the bole

\begin{tabular}{|c|c|c|c|c|}
\hline Entre les caractères & Espèces & Génotypiques & Dues au milieu & Phénotypiques \\
\hline $\begin{array}{l}\text { Densité du bois de cœur } \\
\text { et } \\
\begin{array}{l}\text { Densité du bois périphé- } \\
\text { rique }\end{array}\end{array}$ & P. nigra $\ldots$. & $0,99 * *$ & $-0,17 \mathrm{NS}$ & $0,67 * *$ \\
\hline
\end{tabular}

Les corrélations génotypiques jeune-adulte concernant la densité du bois ont des valeurs élevées $(0,72$ et 0,60 pour Populus nigra et $P$. $x$ euramericana respectivement) qui semblent autoriser une bonne prédiction de la densité et, par conséquent, permettre une sélection efficace dès l'âge d'un an. II n'en est pas de même pour la longueur des fibres dont la corrélation jeune-adulte est plus basse. Ce fait, lié à une faible variabilité, n'autorise pas la sélection juvénile pour ce caractère.

En ce qui concerne le tableau 10, on note également pour les deux espèces une forte liaison d'ordre génétique. En revanche, les corrélations au niveau du milieu sont nulles statistiquement. Pour $P$. nigra et $P$. $x$ euramericana une grande similitude apparaît dans les résultats.

Des calculs analogues à ceux dont les résultats figurent dans le tableau 10 ont été menés pour $P$. euramericana pour la circonférence et la hauteur. On observe des valeurs nulles pour les trois types de corrélations qui nous intéressent. Ceci est très différent des résultats observés pour l'infradensité (on se souviendra cependant des réserves apportées à la signification des mesures de «vigueur » dans le jeune âge).

Notons également que, pour $P$. nigra chez lequel une variabilité génotypique se manifeste à la fois à l'état jeune et à l'état adulte, le retrait tangentiel présente une forte valeur pour $r_{G}$ (quasi égale à 1). Ses deux autres liaisons ne sont significatives qu'à 5 p. 100 (-0,45 pour $r_{E}$, $+0,46$ pour $\left.r_{P}\right)$; l'opposition entre $r_{E}$ ef $r_{G}$ se retrouve pour d'autres essences et d'autres caractères; elle nous montre l'intérêt de la décomposition de la covariance phénotypique.

\section{4. - Corrélations entre caractères}

Le mode de calcul est le même que celui employé pour les corrélations du paragraphe précédent.

\subsection{Corrélations à l'état jeune.}

Le tableau 11 donne les principales valeurs que nous avons calculées. Les caractères non analysés sont ceux qui ne présentaient pas de variabilité clonale. 


\section{TABLEAU 11}

Corrélations entre caractères à l'état juvénile

Correlations between characteristics ; juvénile stages

\begin{tabular}{|c|c|c|c|c|c|c|}
\hline & \multicolumn{2}{|c|}{$\begin{array}{l}\text { Densité du bois des } \\
\text { arbres adultes (moyenne } \\
\text { Est-Ouest ; côté cœur) }\end{array}$} & \multicolumn{2}{|c|}{$\begin{array}{c}\text { Croissance en hauteur juvénile } \\
\text { des arbres adultes }\end{array}$} & \multicolumn{2}{|c|}{$\begin{array}{l}\text { Croissance en circonférence } \\
\text { juvénile des arbres adultes }\end{array}$} \\
\hline & P. nigra & P. $x$ euram. & P. nigra & P. $x$ euram. & P. nigra & P. $x$ euram. \\
\hline $\begin{array}{l}\text { Densité } \\
\text { du bois } \\
\text { des pousses } \\
\text { d'un an }\end{array}$ & $\mathbf{r}_{\mathrm{G}}=0,76^{* *}$ & ${ }^{*} r_{G}=0,54 * *$ & $r_{G}=-0,18 N S$ & $\mathrm{r}_{\mathrm{G}}=-0,03 \mathrm{NS}$ & $\mathrm{r}_{\mathrm{G}}=-0,30 \mathrm{NS}$ & $r_{G}=-0,09 N$ \\
\hline $\begin{array}{l}\text { Densité } \\
\text { du bois } \\
\text { des arbres } \\
\text { adultes } \\
\text { (moyenne est- } \\
\text { ouest; côté } \\
\text { cœur) }\end{array}$ & & 1 & $\begin{array}{l}r_{G}=-0,50 * \\
r_{E}=-0,12 N S \\
r_{P}=-0,74 * *\end{array}$ & $\begin{array}{l}r_{\mathrm{G}}=-0,40 * \\
r_{\mathrm{E}}=-0,10 \mathrm{NS} \\
r_{\mathrm{P}}=-0,34 \mathrm{NS}\end{array}$ & $\begin{array}{l}r_{\mathrm{G}}=-0,62 * * \\
r_{\mathrm{E}}=-0,20 \mathrm{NS} \\
r_{\mathrm{P}}=-0,67 * *\end{array}$ & $S \begin{array}{l}r_{G}-0,13 \mathrm{NS} \\
r_{E}-0,01 \mathrm{NS} \\
r_{P}-0,27 \mathrm{NS}\end{array}$ \\
\hline
\end{tabular}

première valeur $=$ corrélation génotypique $r_{G}$

deuxième valeur $=$ corrélation due à l'environnement $r_{E}$

troisième valeur $=$ corrélation phénotypique $r_{P}$

$* *$ ef $*$ valeurs significatives aux seuils de 1 et $5 \%$ respectivement

\section{Quatre faits ressortent de ce tableau :}

- les corrélations génotypiques entre la densité du bois des pousses d'un an et la densité du bois de cœur des arbres adultes sont élevées. Elles expriment la forte dépendance génétique du caractère ;

- les corrélations génotypiques entre la densité des pousses d'un an et les caractéristiques auxométriques juvéniles des arbres adultes ne diffèrent pas de zéro. La sélection de clones à forte densité destinés à de courtes rotations pour la production de particules ne devrait pas entraîner une perte de volume de matière ligneuse. C'est un résultat rassurant pour le sélectionneur;

- en revanche, les corrélations génotypiques et phénotypiques entre la densité du bois de cœur des arbres adultes et leur croissance juvénile semblent significatives et négatives chez $P$. nigra, alors qu'elles sont beaucoup moins sensibles chez $P$. euramericana. Malgré tous les doutes que nous émettons au sujet de la valeur des observations auxométriques juvéniles, il semblerait que chez Populus nigra, il faudrait peutêtre se méfier des conséquences secondaires d'une sélection au profit de l'un des deux caractères concernés. Cette conclusion va à l'encontre de celle de l'alinéa précédent. Cette distorsion peut s'expliquer par le fait que, dans le cas de l'analyse de variancecovariance pour les arbres adultes de Vineuil, nous obtenons des valeurs qui peuvent être entachées d'un effet «milieu commun». En revanche, les estimations calculées par régression entre bois d'un an à Velaine et bois adulte à Vineuil ne sont pas biaisées systématiquement du fait de l'inexistence de dispositif ; elles peuvent intégrer une éventuelle interaction génotype-environnement et sont, par conséquent, de 
portée plus générale. Nous sommes donc tentés de lui accorder beaucoup plus de crédit ;

- les corrélations environnementales sont nulles, ce qui tendrait à prouver que toute incidence sur le milieu permettant d'augmenter la production en volume (une fertilisation par exemple) ne pourrait avoir d'incidence dépressive sur la densité du bois de ces taillis. In fine la masse de matière produite serait donc accrue. Ceci devrait bien entendu être vérifié expérimentalement.

Notons qu'un calcul effectué sur $P$. nigra pour le couple densité et retrait tangentiel indique de fortes liaisons de même signe pour les trois niveaux considérés $\left(r_{\mathrm{G}}=0,59 * *, r_{\mathrm{E}}=0,51 * *, r_{\mathrm{P}}=0,56 * *\right)$. C'est là un aspect assez négatif ; le populiculteur doit s'attendre à obtenir un matériau qui subira des déformations plus élevées au séchage s'il opte pour une amélioration des propriétés mécaniques grâce à l'augmentation de la densité.

\subsection{Corrélations à l'état adulte.}

Le tableau 12 rassemble les principales valeurs calculées. On vérifie d'abord que chez les peupliers, le couple de torsion semble aussi être un assez bon indicateur de la densité du bois. Ceci paraît surtout vrai pour les peupliers euraméricains mais une tendance similaire se dessine chez les $P$. nigra avec une valeur positive, mais cependant non significative $(0,35)$ au niveau génotypique.

\section{TABLEAU 12}

Corrélations entre caractères à l'état adulte

Correlations between characteristics; Mature stage

\begin{tabular}{|c|c|c|c|c|c|c|c|c|c|c|c|}
\hline & & \multicolumn{2}{|c|}{$\begin{array}{l}\text { Densité } \\
\text { moyenne }\end{array}$} & \multicolumn{2}{|c|}{$\begin{array}{c}\text { Retrait } \\
\text { tangentiel }\end{array}$} & \multicolumn{2}{|c|}{$\begin{array}{l}\text { Longueur } \\
\text { des fibres }\end{array}$} & \multicolumn{2}{|c|}{$\begin{array}{l}\text { Croissance } \\
\text { en hauteur }\end{array}$} & \multicolumn{2}{|c|}{$\begin{array}{c}\text { Croissance } \\
\text { en circonférence }\end{array}$} \\
\hline & & P. nigra & P. euram. & P. nigra & P. euram. & P. nigra & P. euram. & P. nigra & P. euram & $P$. nigra & P. euram. \\
\hline $\begin{array}{l}\text { Couple } \\
\text { de } \\
\text { torsion }\end{array}$ & $\begin{array}{l}r_{G} \\
r_{E} \\
r_{P}\end{array}$ & $\begin{array}{l}0,35 \text { NS } \\
0,58 * * \\
0,41 *\end{array}$ & $\begin{array}{c}0,90 * * \\
-0,05 \mathrm{NS} \\
0,66^{* *}\end{array}$ & $\begin{array}{c}-0,29 \mathrm{NS} \\
0,43^{*} \\
-0,07 \mathrm{NS}\end{array}$ & $\begin{array}{c}0,56 * * \\
-0,38^{*} \\
0,20 \mathrm{NS}\end{array}$ & $\begin{array}{c}\text { non } \\
\text { mesuré }\end{array}$ & $\begin{array}{l}0,48 * * \\
0,00 \text { NS } \\
0,30 \text { NS }\end{array}$ & $\begin{array}{c}-0,51 * \\
0,38 \text { NS } \\
-0,47^{*}\end{array}$ & $\begin{array}{l}0,10 \mathrm{NS} \\
0,25 \mathrm{NS} \\
0,13 \mathrm{NS}\end{array}$ & $\begin{array}{r}-0,16 \mathrm{NS} \\
0,07 \mathrm{NS} \\
-0,23 \mathrm{NS}\end{array}$ & $\begin{array}{l}0,17 \text { NS } \\
0,45^{*} \\
0,14 \text { NS }\end{array}$ \\
\hline $\begin{array}{l}\text { Densité } \\
\text { oyenne } \\
\text { du bois }\end{array}$ & $\begin{array}{l}r_{\mathrm{G}} \\
r_{\mathrm{E}} \\
r_{\mathrm{P}}\end{array}$ & & 1 & $\begin{array}{l}0,55^{* *} \\
0,43^{*} \\
0,50 *\end{array}$ & $\begin{array}{l}0,52 * * \\
0,25 \text { NS } \\
0,39 *\end{array}$ & $\begin{array}{c}\text { non } \\
\text { mesuré }\end{array}$ & $\begin{array}{c}0,68 * * \\
-0,09 \mathrm{NS} \\
0,36 \mathrm{NS}\end{array}$ & $\begin{array}{l}0,25 \mathrm{NS} \\
0,27 \mathrm{NS} \\
0,48^{*}\end{array}$ & $\begin{array}{l}0,02 \mathrm{NS} \\
0,29 \mathrm{NS} \\
0,08 \mathrm{NS}\end{array}$ & $\begin{array}{c}-0,25 \mathrm{NS} \\
0,51 * * \\
0,12 \mathrm{NS}\end{array}$ & $\begin{array}{r}0,20 \mathrm{NS} \\
-0,06 \mathrm{NS} \\
0,15 \mathrm{NS}\end{array}$ \\
\hline
\end{tabular}

A noter ensuite la relation positive (pour $r_{G}$ ) entre densité et longueur des fibres qui ne prête pas à conséquence étant donné la faible variabilité clonale du deuxième caractère (cf. $\S 4.23$ ).

On remarque enfin et surtout chez $P$. euramericana la quasi-indépendance génétique entre densité et croissance, aussi bien aux niveaux génétique, environnemental que phénotypique. Les liaisons retrait tangentiel-couple ou retrait tangentiel-infradensité sont parfois significatives; il semblerait que l'amélioration de la densité par 
voie génétique entraîne une hausse corrélative du retrait tangentiel. Nous rejoignons en cela une des conclusions d'ordre technologique du point précédent. Les choses semblent moins nettes au niveau du milieu.

\section{5. - Conclusion}

Aux questions posées en introduction, il est possible de répondre de la façon suivante :

\section{- Existence et cause d'une variabilité intraclone}

Une certaine variabilité des caractéristiques de la qualité du bois peut être attribuée à des causes non génétiques qui sont principalement :

- les vents dominants qui provoquent vraisemblablement du bois de tension chez certains clones euraméricains ;

- le vieillissement de l'arbre qui produit une variation de la densité sans que cette variation soit systématiquement une augmentation comme pour la plupart des essences étudiées jusqu'ici.

- Existence d'une variabilité génétique aux stades juvénile ef adulte

Cette variabilité existe pour certains caractères étudiés. Ceux-ci sont principalement :

- l'infradensité

- le retrait tangentiel

- le rendement en fibres

- la croissance

Mais d'une manière générale, les caractéristiques de la qualité du bois ont des amplitudes de variation assez faibles dans l'échantillonnage que nous avons étudié. Les gains génétiques possibles, malgré certaines héritabilités au sens large assez élevées, sont de ce fait relativement faibles.

- 6 p. 100 pour la densité chez $P$. euramericana

- 3 p. 100 pour la longueur des fibres contre

- 40 p. 100 pour la croissance en hauteur des arbres adultes

- 63 p. 100 pour leur croissance en circonférence.

Il pourrait donc être considéré comme illusoire de chercher à profiter d'un gain aussi faible. Eł pourtant les faits sont là ; I 214 et Gelrica n'ont pas des caractéristiques de leur bois entièrement satisfaisantes. Et il n'est pas improbable qu'une augmentation de 9 p. 100 de la densité (meilleur clone euraméricain par rapport à la moyenne) et 24 p. 100 (par rapport au moins bon clone) produise de profondes modifications dans la structure du bois qui améliore ses qualités dans de larges proportions. Des conclusions analogues ont été trouvées pour un échantillon de dix clones de Populus trichocarpa par Reck (1974) en Allemagne. 
Remarquons à ce sujet que les gains sur la densité apparaissent ici sensiblement supérieurs à ceux que l'on peut attendre pour le même caractère chez les conifères.

\section{- Existence d'une corrélation jeune-adulte}

Une corrélation génotypique jeune-adulte très marquée semble exister pour la densité du bois des deux types de peupliers que nous avons étudiés. Cela autorise des possibilités de sélection, dès la pépinière, pour cette caractéristique très importante de la qualité du bois. La faiblesse du gain potentiel devrait inciter à une forte intensité de sélection. La valeur des coefficients de corrélation relativement éloignée de 1 doit rendre le sélectionneur prudent (longueur de fibres).

\section{- Existence de corrélations génotypiques entre caractères}

Le choix de clones ayant un bois à haute densité n'aura pas de conséquences indirectes notoires. II apportera cependant :

- un retrait tangentiel élevé. On observe par exemple sur $P$. euramericana une densité de $355 \mathrm{~g} / \mathrm{dm}^{3}$ pour Robusta et de $287 \mathrm{~g} / \mathrm{dm}^{3}$ pour I 214 (côté écorce). Entre ces deux clones la différence entre les retraits tangentiels est de 1,7 p. 100 (8,1 p. 100 pour Robusta, 6,4 p. 100 pour I 214). Il faut donc que l'améliorateur soit conscient qu'un gain appréciable en densité pourra entraîner des déformations plus importantes des feuilles de déroulage,

- des fibres plus longues, quoique le caractère semble peu variable.

Ce choix n'aura, de plus, pratiquement aucun effet sur la croissance. Concrètement, on pourra sélectionner des peupliers euraméricains dont le bois a une densité élevée, sans perdre sur la croissance. Par la suite, les corrélations densité-croissance étant nulles statistiquement, on est autorisé à penser qu'il est possible d'augmenter la production en volume par fertilisation sans risquer de voir diminuer la densité.

Naturellement, il serait judicieux, avant de tirer des conclusions par trop rapides du coefficient $r_{E}$ observé ici, d'effectuer des tests clonaux combinés à des essais d'apport d'engrais afin de disposer d'une gamme étendue de fertilités.

La solution de ce problème est vraisemblablement le choix d'individus ayant un bois dense et une forte productivité. Mais, si la recherche de cette haute densité est possible dès le jeune âge, la productivité semble n'être dotée d'une liaison génotypique juvénile-adulte étroite que dans le cas d'espèces pures : Populus deltoïdes et $P$. nigra, alors qu'aucune corrélation n'existerait pour des hybrides tels que les peupliers euraméricains (Sekawin, 1972). II n'est pas inutile de rappeler dans cette conclusion la prudence avec laquelle il est cependant nécessaire de considérer les résultats donnés ici, faute de l'existence de dispositifs expérimentaux.

Retenons de tout ceci la possibilité d'un test précoce concernant la densité du bois, son absence de conséquence sur la production en volume. Notons enfin qu'une étude analogue sera entreprise sur $P$. trichocarpa, espèce prometteuse en matière de populiculture forestière. 


\section{Remerciements}

Nous exprimons notre gratitude au Centre Technique du Génie Rural des Eaux et des Forêts qui a mis à notre disposition le Populetum de Bourret. Nous remercions le personnel de la Station de Recherches sur la Qualité des Bois, en particulier celui du Laboratoire de papeterie ainsi que Madame Aubert qui ont réalisé l'importante quantité de prise de données que représente cette étude ainsi qu'une partie de son interprétation statistique.

\section{Summary}

\section{Juvenile selection for wood quality in Populus nigra and Populus euramericana}

The question is raised whether wood basic density in poplars is a characteristic which can be included in early selections. In quantitative genetic terms this can be turned into three questions: 1) what is the magnitude of the variability of wood quality characteristics in poplars;2) how high are the genotypic correlation coefficients between one year old and mature trees for different characteristics of the wood quality ; 3) consequently what secondary effects would arise after a selection for any wood quality characteristic either on other characteristics of the wood or on any other economic characteristic.

Twenty three clones of Populus nigra $L$. and twenty eight clones of $P$. $x$ euramericana (Dode) Guinier were chosen. The one year old stems were cultivated in a nursery near Nancy (Meurthe-et-Moselle), the mature trees belong to the Vineuil National Populetum near Blois (Loir-et-Cher). They are approximately twenty years old. Although each clone (ortet) was replicated twice, both near Nancy and in Vineuil, the two replicates (ramets) grew near each other. Due to "common effects " some of the genetic parameters that have been calculated might be under - or overestimated. For instance, the intraclonal variances being minimized, the broad sense heritabilities might be overestimated.

The characteristics of wood quality assessed in this study were measured directly on one year old stems without bark and on increment cores taken both on the weather and lee-sides of the bole of mature trees. They are : basic density, shrinkage, fiber length and yield to which were added girth and height increments.

The results show that : 1) Basic density, tangentiel shrinkage, fiber yield and of course increment are submitted to clonal variation. The magnitude of wood characteristics variability is low and gives genetics gain of 6 p. 100 for basic density and 2 p. 100 for fiber yield compared to gains of 40 and 63 p. 100 for height and girth increments of mature trees. Although rather low, a gain of 6 p. 100 on basic density seems to have great consequences on wood resistance ; 2 ) The values of the juvenile mature genotypic correlations for basic density $(0,72 * *$ for $P$. nigra and $0.60 * *$ for $P$. $x$ euramericana) are high and show a strong genetic domination of this characteristic which would enable the tree breeder to select clones with a high wood density in the early stages of their life ; 3) Such a selection added to the significant values of genotypic correlations would lead to an unfavorable and rather high tangential shrinkage (maximum values 8,1 p. 100 for Robusta, 6,4 p. 100 for I 214). On the contrary the absence of correlation between density and increment would lead to no disavantageous effects of the selection for wood density.

\section{Zusammenfassung}

\section{Jugendliche Auslese für die Holzqualität in gewissen Schwarzpappeln}

23 Klonen von Populus nigra L. und 28 von P. x euramericana (Dode) Guinier wurden Versuchen unterworfen, um die Möglichkeit zur frühen Auslese der Pappeln für die Holzqualität klarzustellen. Die Beobachtungen betrafen im Jugendstadium einjährige entrindeten Stengelstücke zu zwei pro Klone und zum erwachsenen Stadium etwa 20 Jahre alten Baumbohrspäne der Sammlung des nationalen Populefum von Vineuil nahe bei Blois (2 pro Klone).

Das Vorliegen einer Variabilität für gewisse Eigenschaften ergibł die Möglichkeit, einen genetischen Gewinn zum Beispiel für die Holzrodichte $(6 \%$ ) zu nützen. Die genotypischen Korrelationen zwischen jungen und erwachsenen Bäumen für die Holzdichte scheinen eine Auslese für diese Eigenschaft schon am ersten Jahr zu ermöglichen. Es wurde keinen Verlust in der Produktion in Volumen bewirken, weil es keine Korrelation zwischen Dichte und Wachstum gibt. Im Gegenteil konnte es zu einer indirekten Auslese von Klonen mit hoher Tangentialschwindung führen. 


\section{Références bibliographiques}

CENTRE TECHNIQUE DU BOIS, Paris, 1957. Etude du bois de quelques types de peupliers largement cultivés en France. Actes du VIe Congrès de la Commission Internationale du Peuplier, Paris, 365-400.

CLEMENT et JANIN, 1972. Mise en évidence de cristaux de carbonate de calcium dans le bois des peupliers. Conséquence sur la répartition des ions minéraux liée à la duraminisation CNRF, Station de Recherches sur la Qualité des Bois. Annales des Sciences Forestières, 29 (1), 67-105.

JANIN G., 1972. Microcuissons papetières. La Papeterie, 3, 174-188.

JANIN G., 1973. Détermination des diagrammes polaires des rendements et des indices de permanganate sur carottes droites et obliques. Essais de microclassage sur carottes obliques. CNRF, Station de Recherches sur la Qualité des Bois, Doc. n 1973/4.

NEPVEU G., 1973. Etude génétique de quelques qualités du bois de pin maritime. Corrélations avec des caractères de croissance et d'aptitude à l'élagage. CNRF, Station de Recherches sur la Qualité des Bois. Document à distribution limitée 1973/5, 44 p.

NEPVEU G., et TEISSIER du CROS E, 1976. Sélection juvénile pour la qualité du bois chez les peupliers euraméricains. IUFRO Réunion des Groupes de Travail de Génétique S.2.04.01 à 03 et 05. Bordeaux-France, 14/16 juin 1976, 38 p. Résumé anglais de 7 pages.

POLGE H., 1963. Contribution à l'étude de la qualité du bois des principales essences résineuses exotiques utilisées dans les reboisements français. Annales de l'Ecole Nationale des Eaux et Forêts ef de la Station de Recherches et Expériences, tome XX, fascicule 3.

POLGE H., 1964. Le bois juvénile des conifères. Revue Forestière Française, $\mathrm{n}^{\circ} 6$, juin.

POLGE H. ef ILLY G., 1963 ou 1967. Observations sur l'anisotropie du pin maritime des Landes Ann. Sci. For., 24 (3), 205-231.

POLGE H., et KELLER R., 1970. Première appréciation de la qualité du bois en forêt par utilisation d'un torsiomètre. Ann. Sci. For., 27 (2), 197-223.

POLGE H., 1973. Rapport de Mission. F. A. O. Commission Internationale du Peuplier. Réunion du Groupe de Travail de l'Exploitation et de l'Utilisation du bois. 4 p.

POLGE H., KELLER R., et THIERCELIN F., 1973. Influence de l'élagage de branches vivantes sur la structure des accroissements annuels et sur quelques caractéristiques du bois de Douglas et de Grandis. Ann. Sci. For., 30 (2), 127-140.

RECK S. G., 1974. Growth and wood characteristics in clones of Populus trichocarpa grown in West Germany and their significance for selection. Proceedings Joint IUFRO Meeting Forest Genetics Stockholm, pp. 129-143.

SEKAWIN M., 1972. La sélection précoce des peupliers vis à vis de l'accroissement. Cellulosa e Carta, 23 (8), 35-44.

TEISSIER DU CROS E., KELLER R., 1973. Détermination précoce de certaines caractéristiques du bois de Populus nigra L. C.N.R.F., Station d'Amélioration des Arbres Forestiers, Document à distribution limitée $n^{0}$ 1973/1. Station de Recherches sur la Qualité des Bois. Document à distribution limitée $\mathrm{n}^{0} 1973 / 2$. 
ANNEXE

\begin{tabular}{|c|c|c|c|c|}
\hline \multicolumn{3}{|c|}{ Populus nigra } & \multicolumn{2}{|c|}{ Populus euramericana } \\
\hline Nom (Name) & Origine (Origin) & $\begin{array}{c}\text { Abré- } \\
\text { viation }\end{array}$ & Nom (Name) & $\begin{array}{l}\text { Abré- } \\
\text { viation }\end{array}$ \\
\hline $\begin{array}{l}\text { Bourgoin 51/1 } \\
\text { Illard } 2 \\
\text { Illard } 5 \\
6 / 1 \\
6 / 2 \\
17 \\
\text { Saint Vallier } 2 \\
\text { Saint Vallier } 4 \\
\text { Piboulette } 6 \\
\text { Fastigié } \\
\text { Cazebonne } 4 \\
\text { Blanc de Ga- } \\
\quad \text { ronne } \\
\text { Vert de Ga- } \\
\quad \text { ronne } \\
\\
\text { Veuves } 2 \\
\text { Veuves } 5 \\
\text { V } 6 \\
\text { S } 32 \\
\text { R } 5 \\
\text { Dode } \\
\text { Italica } \\
\text { Carrière bleue } \\
\text { Gigantea } \\
\text { Cranwich }\end{array}$ & 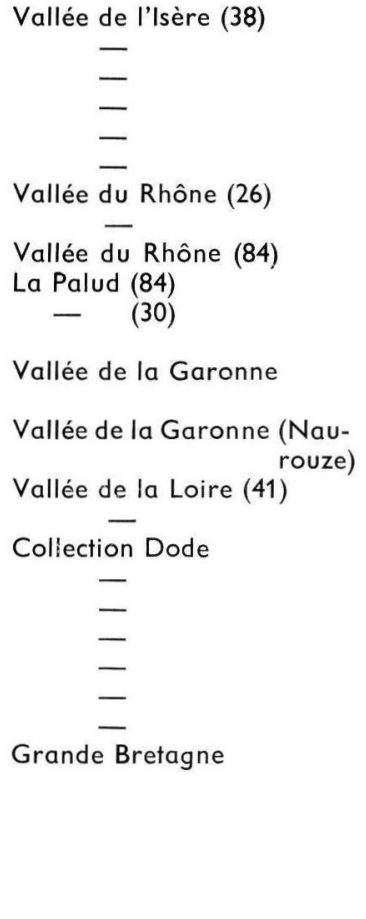 & $\begin{array}{l}\text { B 51/1 } \\
\text { ILR } 2 \\
\text { ILR } 5 \\
6 / 1 \\
6 / 2 \\
17 \\
\text { SVL } 2 \\
\text { SVL } 4 \\
\text { PBL } 6 \\
\text { FST } \\
\text { CZB } 4\end{array}$ & $\begin{array}{l}\text { Veuves } 1 \\
\text { Culasso } \\
\text { Sélys } \\
\text { Ourcq } 2 \\
\text { Ourcq } 4 \\
\text { Jameyzieu } \\
\text { La Font Vert } 9 / 60 \\
\text { Dode } \times 9 \\
\text { D } 363 \\
\text { Touretensis } \\
\text { Bâtard de Hauterive } \\
\text { Saint Marcel } \\
\text { Ottenheim P } 3 \\
\text { La Font Vert } 8 \\
\text { S } 29\end{array}$ & $\begin{array}{l}\text { VVS } 1 \\
\text { CLS } \\
\text { SLS } \\
\text { ORQ } 2 \\
\text { ORQ } 4 \\
\text { JMZ } \\
\text { LFV } 9 / 60 \\
\text { X } 9 \\
\text { D } 363 \\
\text { TRT } \\
\text { BHT } \\
\text { SMR } \\
\text { OTH P3 } \\
\text { LFV } 8 \\
\text { S } 29\end{array}$ \\
\hline
\end{tabular}

